\title{
La mise en valeur agricole du Marais-Vernier
}

\section{The agricultural development of the Vernier Marsh}

\author{
PAR Y. FERRONNIERE \\ INGÉNIEUR A LA COMPAGNIE DES TECHNIQUES HYDHAULIQUES ET AGRICOIFS \\ ET A La SOCIÉTÉ D'ANÉNAGEMENT RURAL ET URBAIN
}

\begin{abstract}
Situation géographique, cadre agricole et hydraulique, histoire des aménagements antérieurs et de leurs échecs. - Reprise récente des travaux d'assainissement par l'amélioration de l'émissaire et de son réglage; travaux de défrichement, leurs difficultés; essais de mise en culture, leurs conclusions souvent négatives; premiers succès remportés dans le domaine de l'élevage; la valeur de l'expérience acquise.
\end{abstract}

\begin{abstract}
Geographical location, agricultural and hydralic framemork, history of previons improvements and their setubacks. Recent renewal of drainage work by improving the emissary and regulating it; land clearing work, its difficullies; crop establishment tests, their conclusions often negative; first successes achieved in stock raising; the value of the experience acquired.
\end{abstract}

\section{Situation}

Le Marais-Vernier est situé en Normandie, à l'extrémité nord du département de l'Eure, à proximité immédiate de l'estuaire de la Seine et sur sa rive gauche, à quelques kilomètres de

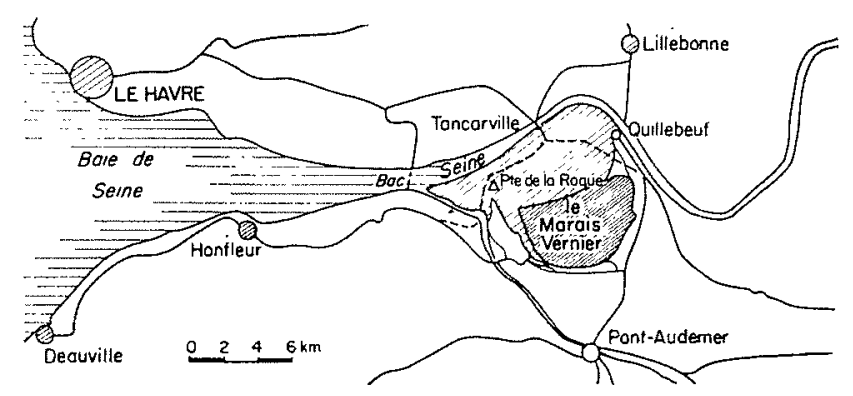

Fri, 1, .... Plan de situation.

Quillebeuf. II s'étend sur 2.200 hectares, dans une cuvette semi-elliptique creusée dans la craic du plateau du Roumois par un ancien méandre du fleuve. Depuis l'abandon de cette ancienne partie de son lit, le cours de la Seine s'est éloigné de plusieurs kilomètres vers le nord, constituant une large zone d'alluvions; une digue naturelle, renforcée au $\mathrm{xvIr}^{\mathrm{e}}$ siècle, sépare ainsi nettement les alluvions de la cuvette tourbeuse du Marais-Vernier.

Il semble que dès le vi ${ }^{*}$ siccle les moines de l'Abbaye de Jumièges aient commencé l'assainissement du Marais. En 1617, le Hollandais Humfroy BRADLEY s'engageait à achever les travaux de desséchement en trois ans. En 1847 enfin, par ordonnance royale, les propriétaires du MaraisVernier étaient réunis en syndicat «pour perfectionner et entretenir le desséchement dont il s'agit $\gg$.

Ainsi, à trois reprises au moins, des travaux hydrauliques ont été exéculés au Marais-Vernier. Ceux de 1847-1856 sont assez bien connus : ils ont porté surtout sur l'exutoire, canal de SaintAubin, et son débouché en Seine. Le radier en fut abaissé; mais en deux zones, vers le Pont des Tiquets et vers Saint-Aubin, les travaux manuels furent contrariés par la dureté des couches rencontrées que les outils à main ne pouvaient entamer. L'exutoire était ainsi établi à une cote trop élevée et sans débit suffisant; après une période d'efficacité relative, les travaux, mal entretenus, s'avérèrent à nouveau toul $\dot{a}$ fait inefficaces et le marais redevint inculte sur sa plus grande surface. 


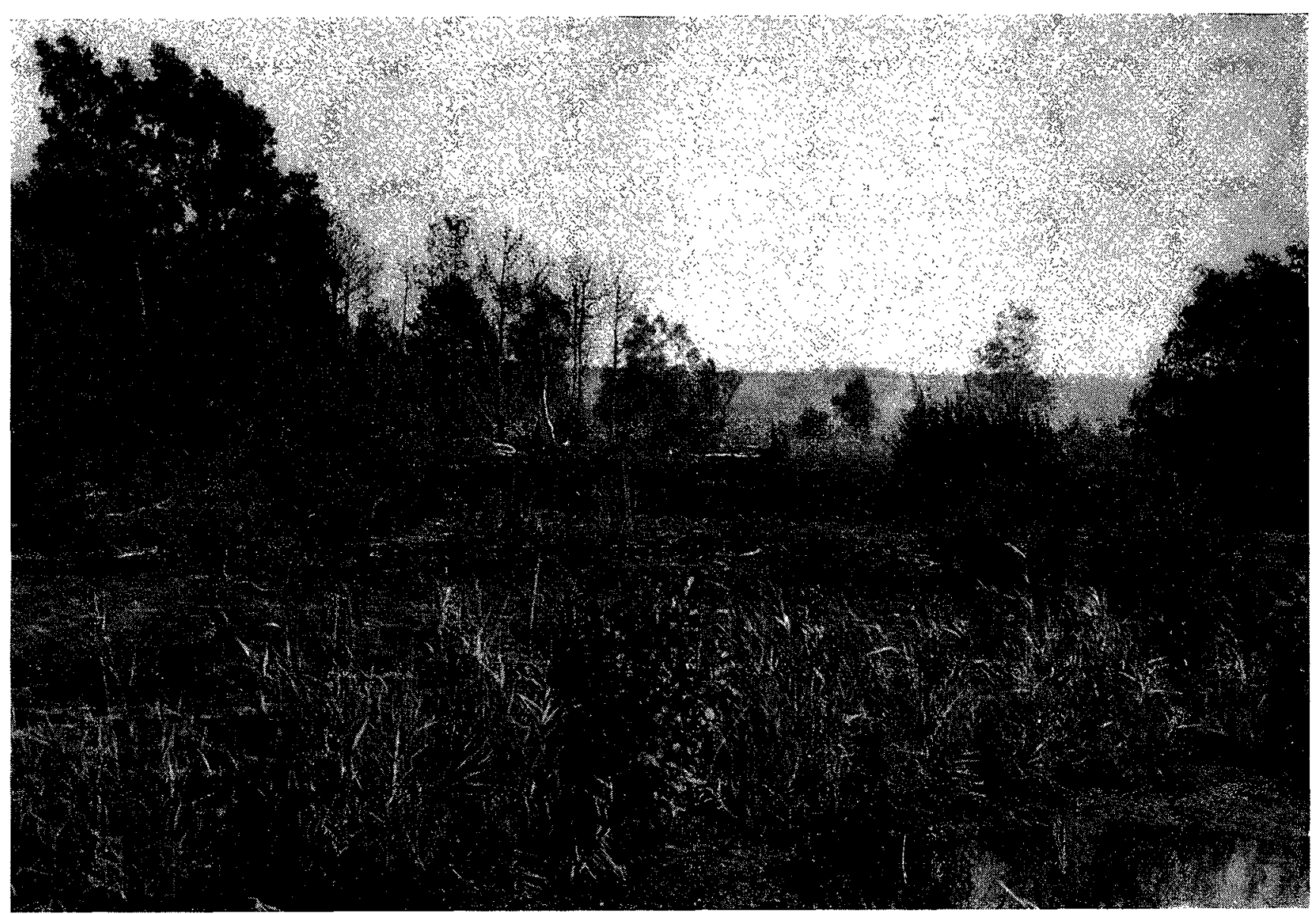

Fig. 2. - L'état chaotique du marais avant défrichement.

Vers les années 1940 , le Marais-Vernier se présentait comme une vaste étendue plane (aux cotes $+2,10 \mathrm{~m} \mathrm{N.G.F.} \mathrm{au} \mathrm{centre,} \mathrm{et}+2,60 \mathrm{~m}$ sur les bords), coupée par quelques fossés syndicaux aboutissant à une Grand-Mare naturelle d'où le collecteur, sur $6 \mathrm{~km}$ de long, devait conduire à la Seine les eaux du bassin versant et de la cuvette elle-même, avec une pente qui n'était alors que de $10 \mathrm{~cm}$ par $\mathrm{km}$ et avec une section très insuffisante. Le radier de l'écluse de Quillebeuf se trouvait à la cote $+0,60$ N.G.F., alors que les basses mers de morte-eau descendent à - 1,40 et que les hautes mers de vive-eau s'élèvent à $+3,70$ N.G.F.

Dans le marais même, on distingue trois zones bien distinctes:

- La périphérie, 500 hectares, caractérisée à l'ouest et au sud par de très nombreux fossés radiaux formant limites de propriétés entre une centaine de « courtils», dont la partie haute, avec chaumière et cour de pommiers, restait toujours hors d'eau, tandis que la partie basse, mauvais pré à roseaux et jones, ne pouvait être fauchée comme litière que deux ans sur trois.

Au nord, à l'est et au sud-est, les terres de ceinture du marais étaient aussi toutes exploitées en médiocres prés.

- La partie centrale, 1.100 hectares, constituait une zone inexploitable réservée à la chasse; partout des roseaux, carex, molinies formant «têtes de chien », taillis d'aulnes et de bouleaux avec pins lacustres et même quelques chênes; de rares fossés, envasés; un aspect de sauvagerie et de désolation.

- Entre ces deux parties, une zone intermédiaire de 600 hectares autrefois exploitée mais ne fournissant plus qu'une litière occasionnelle pour le bétail.

En fin avril 1944, plus de 1.500 hectares étaient encore couverts de 20 à $30 \mathrm{~cm}$ d'eau qui ne furent entièrement résorbés qu'au début de juillet.

C'est alors qu'à la suite d'une vente par lots portant sur la moitié de la superficie maréca- 


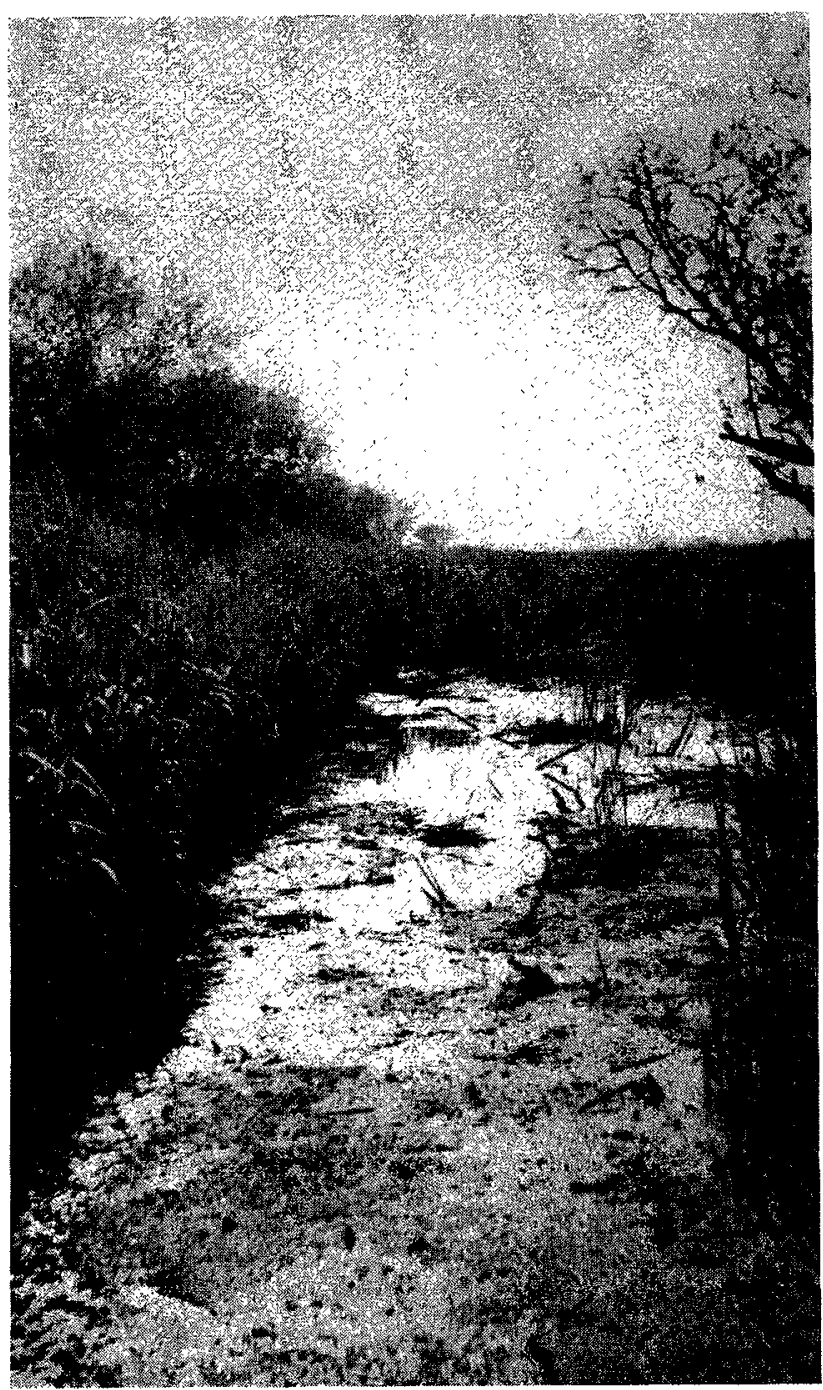

Fig. 3. - L'ancien collecteur vers le débouché de la Grande Mare.

geuse, le Syndicat fut ranimé par quelques-uns des nouveaux propriétaires. Avec le concours du Génie Rural, une étude hydraulique fut confiée aux Etablissements Neyrpic, de Grenoble.

\section{Données de l'étude}

Bassin versant. - Il est composé, outre le marais lui-même, par une partie de la falaise crayeuse qui le borde et qui contient une nappe d'eau souterraine, alimentant les puits régionaux et affleurant dans le marais par de nombreuses sources en général à faible débit (au total $350 \mathrm{l} / \mathrm{s}$ en moyenne).

Topographie. - Large marais bas et sensiblement horizontal dont les cotes d'altitude s'échelonnent entre $+2,10$ et $+2,60 \mathrm{~m}$ N.G.F. avec quelques points vers $+3,50$ sur le pourtour nord-est.

GÉologre. - Le marais est presque uniquement composé de tourbe épaisse de 6 à $8 \mathrm{~m}$, sauf vers le nord-est constitué d'alluvions fluviales.

Cuimat. - A tendance pluviale océanique, caractérisé par des écarts de températures extrêmes relativement faibles et des maxima de précipitations en automne et en hiver - soit 80 a 150 jours de pluie par an et une moyenne annuelle (à Pont-Audemer) de $700 \mathrm{~mm}$ pour la période 1851-1900; en année exceptionnelle, la pluviosité peut atteindre $1.000 \mathrm{~mm}$ ou ne pas dépasser $550 \mathrm{~mm}$. On a observé des maxima de pluies de $118,3 \mathrm{~mm}$ en 9 jours et même $199,3 \mathrm{~mm}$ en 19 jours (novembre 1941).

L'évaporation peut être très importante en śté; elle est pratiquement nulle, du fait de l'état hygrométrique de l'air, pendant la période des srosses précipitations d'hiver.

Régime de la Seine. - La Seine est un fleuve à marées dont l'amplitude, à Quillebeut', atten $3,24 \mathrm{~m}$ (marées de coefficient 45 ); 4,25 m (coefficient 70 ), $5,76 \mathrm{~m}$ (coefficient 100 ) el $6.08 \mathrm{~m}$ (coefficient 120).

Par des observations en plusieurs points de

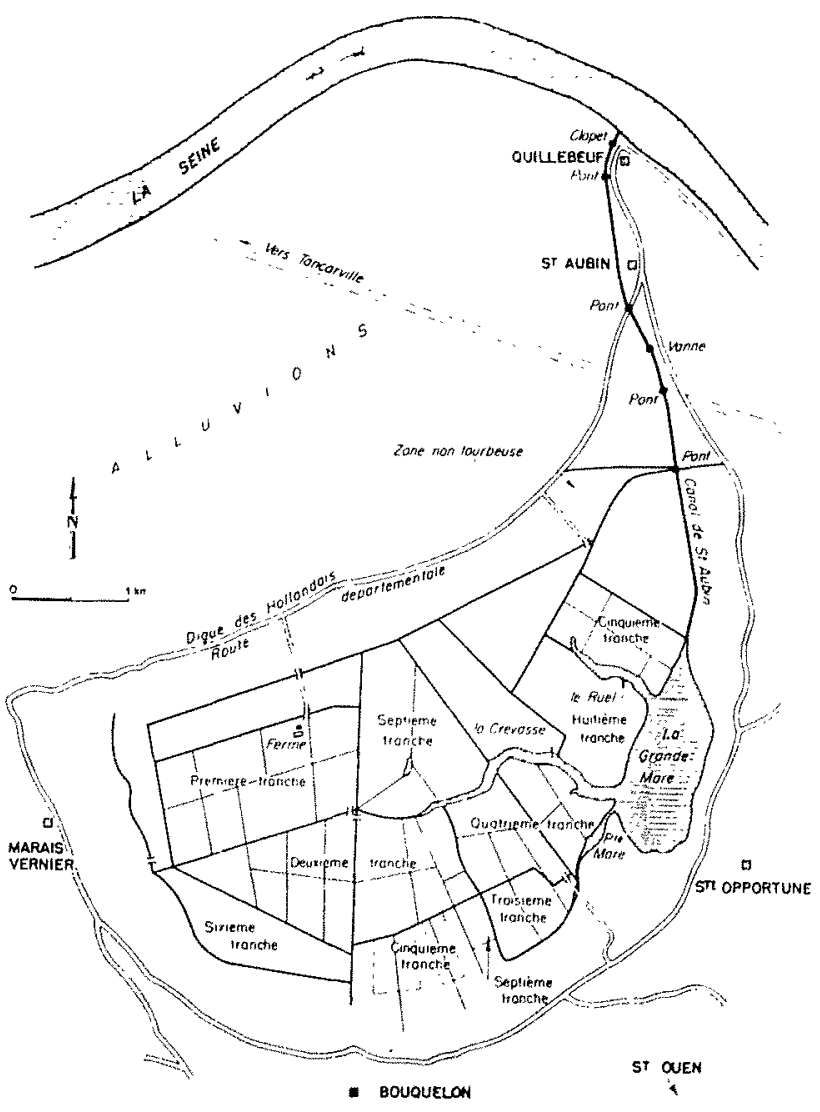

Fri. 4. - Travaux d'assainissement général. 
l'estuaire, il a été constaté que le niveau de la Seine reste à une cote inférieure à $+1,00$ N.G.F. pendant 105 à 120 heures en 15 jours consécutifs et que le chiffre le plus élevé, soit $120 \mathrm{~h}$, correspondait à la zone amont de la cuvette, soit à Quillebeuf; ainsi il y avait intérêt à reprendre l'évacuation des eaux du marais en suivant. le tracé de l'ancien collecteur.

La salinité et la teneur en vase des eaux de Seine à Quillebeuf reste faible, sauf à la fin du flux en marée de vive-eau ( $7 \mathrm{~g}$ de chlore par litre à la haute mer de coefficient 100).

Niveaux de l'eau a maintenir dans le marais.

- En accord avec le Syndicat des propriétaires, il a paru nécessaire de concevoir le réseau d'assainissement de façon que le niveau de l'eau soit à $0,80 \mathrm{~m}$ sous la surface du sol pendant les mois d'hiver et seulement à 0,40 pendant les mois d'été, e'est-à-dire réglable entre les cotes $+1,40$ et 1,80 N.G.F. Ces cotes, relativement hautes, ont été choisies de façon à lutter contre les tassements et à maintenir de la fraîcheur dans le sol en été, donc à empêcher toute dessiccation irréversible de la tourbe.

\section{Assainissement général}

Les travaux d'assainissement, agréés et sub- ventionnés par le Ministère de l'Agriculture, furent réalisés de 1947 à 1950 . Ils ont porté sur les points suivants $\left(^{\star}\right)$ :

$1^{\circ}$ Mise en état du canal de Saint-Aubin, collecteur des eaux, lui permettant d'évacuer $5.800 \mathrm{l} / \mathrm{s}$ (au lieu de $500 \mathrm{l} / \mathrm{s}$, mesurés en 1945 pour un niveau de $+2,10$ dans la Grand'Mare), soit :

Section amont: largeur $12 \mathrm{~m}$, profondeur $2,70 \mathrm{~m}$, profil rectangulaire (terrain tourbeux).

Section médiane jusqu'à la vanne automatique : largeur au plafond $10 \mathrm{~m}$, profondeur moyenne $4,30 \mathrm{~m}$ - talus à $45^{\circ}$ (terrain alluvial).

Section aval: de la vanne automatique au clapet à marées - largeur au plafond $8 \mathrm{~m}$ profondeur supérieure à $5 \mathrm{~m}$ dont plus de $1 \mathrm{~m}$ creusé dans le banc de craie - talus à $45^{\circ}$.

(*) Consulter en particulier : « L'Assainissement du Marais-Vernier », par G. Monand, ingénieur en chef du Génie Rural et A. Bernard, ingénieur des T.R. (le Génie Rural, décembre 1950) et « l'Aménagement hydraulique du Marais-Vernier », par J. Desforges (l'Eau, octobre $1950)$.

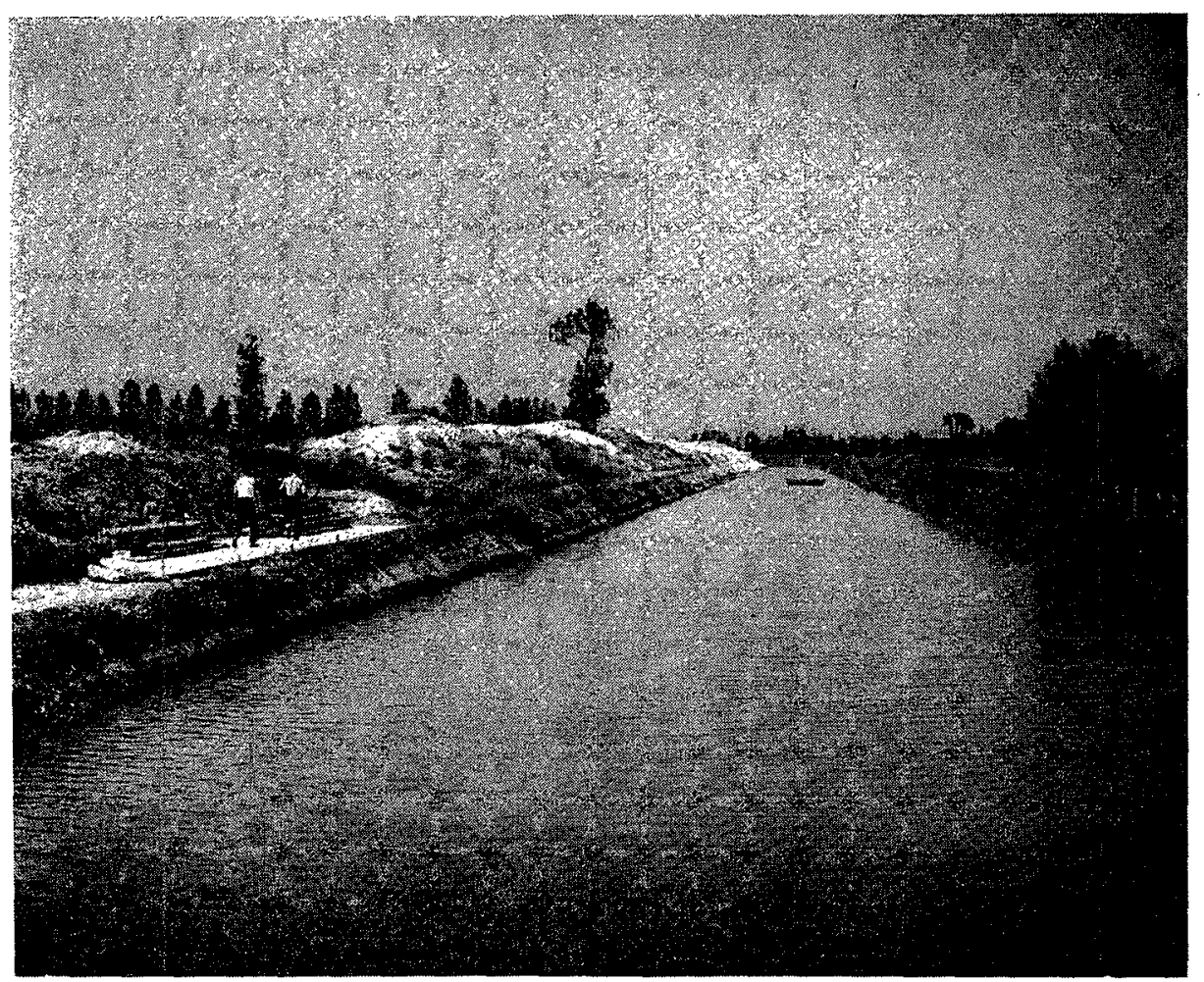

Frg. 5. - Le canal de Saint-Aubin, section médiane. 




Fic. 6. -- Vanne automatique à niveau amont constant.

La pente moyenne de ce collecteur est de $0,2 \mathrm{~mm}$ par mètre.

$2^{\circ}$ L'équipement de ce collecteur par une vanne à niveau amont constant, réglable suivant les saisons de la cote $+1,40$ N.G.F., cote d'hiver, à $+1,80$, cote d'été, résultant d'irrigation à partir d'eau de Seine. Cette vanne commande d'autant mieux le niveau des eaux dans le marais qu'elle est placée plus à l'amont. Mais le mouvement des marées à l'aval de la vanne, avec ses risques d'érosion ou d'envasement ainsi que de remontées d'eau salée, a conduit à placer au débouché en Seine de ce même collecteur un clapet automatique à marées, qui joue en même temps le rôle de vanne à niveau amont constant afin d'éviter qu'un asséchement de la section aval du canal n'épuise la nappe des puits de Quillebeuf. La vanne et le clapet ont été étudiés et fournis par les Etablissements Neyrpic de Grenoble.

$3^{\circ}$ La réfection d'un réseau de $35 \mathrm{~km}$ de fossés syndicaux de $3,50 \mathrm{~m}$ à $6 \mathrm{~m}$ de large et $1,60 \mathrm{~m}$ de profondeur moyenne, presque tous établis en terrain de tourbe.

\section{$4^{\circ}$ La reconstruction de quelques ponts.}

Depuis la pose de la vanne automatique et l'achèvement des travaux, les niveaux d'eau dans le marais ont pu, quelles que soient les circonstances, être réglés à $10 \mathrm{~cm}$ près aux cotes prévues suivant les saisons.
Les précipitations ont dépassé $1.000 \mathrm{~mm}$ au cours de la période $1^{\text {er }}$ juillet $1950-30$ juin 1951 , et atteint $420 \mathrm{~mm}$ d'ean au cours des quatre der" nier's mois de l'année 1952, sans qu'il y ait eu risque d'inondation du Marais-Vernier, alors que toutes les vallées voisines étaient ennoyées.

Par contre, chaque année, le niveau haut d'été a été rétabli par les admissions d'eau de Seine, en période de fortes marées, la salinité restanl insignifiante presque jusqu'à la fin du flot.

L'aménagement hydraulique général du Marais-Vernier a done été tout à fait efficace. La régulation du niveau d'eau, condition nécessaire, permet d'envisager la mise en valeur qui exige des travaux d'une loute autre nature.

\section{Préparation des terres}

Comment, de celte vaste tourbière maintenant hors d'eau, allait-on faire des champs ou des prairies? Le problème était entièrement nouveau en France où, depuis plus de 200 ans, rien de comparable n'avait été fait, sinon sur de toutes petites surfaces. Des travaux d'assainissement analogues à ceux du Marais-Vernier, dans d'autres régions, et sous d'autres conditions hydrauliques, avaient buté sur cet inconnu et avaient laissé en friche des terrains assainis. L'expérience hollandaise en la matière était déjà ancienne et présentait de grandes différences dans les épaisseurs de tourbe, leur composition, 


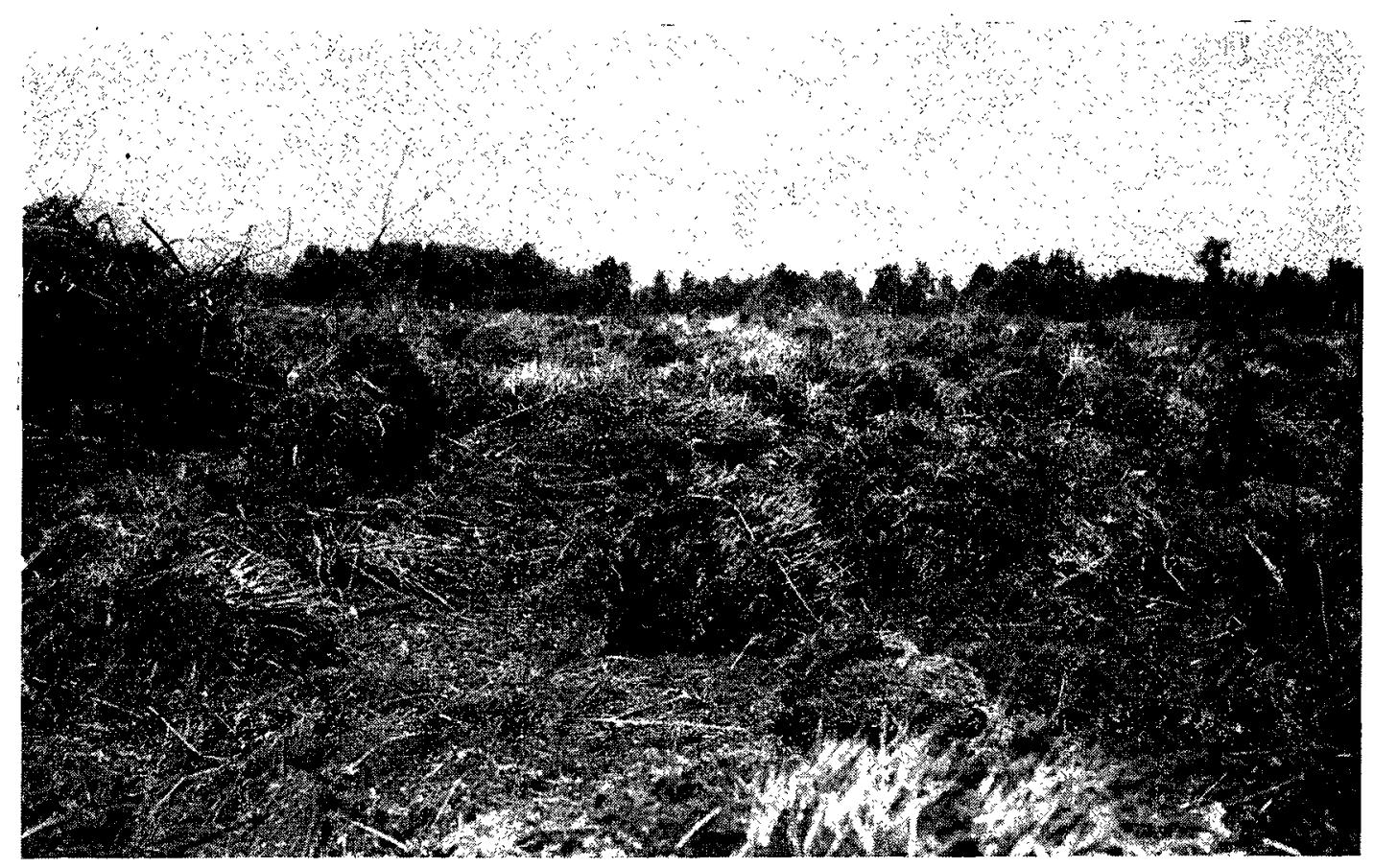

Fic. 7. - La destruction des têtes de chien.

le climat et les conditions de travail (travail en général manuel pour la résorption du chômage).

Tout était donc à innover en la matière.

C'est beaucoup par tâtonnements, essais puis démonstrations qu'une méthode de travail et des résultats satisfaisants ont pu finalement être acquis, au prix d'une ténacité remarquable et d'une farouche volonté d'aboutir.

\section{DéFrichement.}

La mise en valeur a été entreprise au printemps 1949 sur des zones alors complètement incultes.

La végétation naturelle apporte une aide pré-



FIG. 8. - Taillis. cieuse à la mise en route des travaux; elle permet la circulation des tracteurs que la portance initiale de la tourbe nue - $200 \mathrm{~g} / \mathrm{cm}^{2}$ - n'aurait pu admettre.

La tourbe en place se présente en effet la plupart du temps, sous les premiers centimètres d'apparence encore végétale, comme une substance colloïdale molle, brune ou noirâtre, spongieuse et fibreuse parfois, ou bien pâteuse; dans tous les cas elle contient une très forte proportion d'eau de constitution et de rétention (90 à $95 \%$ ); par évaporation elle perd celle-ci et s'affermit alors, mais non celle-là.

Dès que, du fait des intempéries, la teneur en eau de la tourbe mise à nu s'accroît, l'aspect pâteux et inconsistant reparaît; elle est alors presque imperméable, le drainage n'évacuant à peu près que les eaux de ruissellement; sa portance devient très faible, la circulation et le travail des tracteurs deviennent difficiles. Au cours des trois premiers hivers, tous les tracteurs $\left(^{*}\right)$ s'enlisaient au moins une fois par jour, parfois en même temps. Il s'est cependant trouvé une entreprise pour exécuter ces travaux de défrichement : la Société d'Aménagement Rural et Urbain; mieux valait une Société nouvelle, dirigée par un ingénieur agricole ayant le sens et le respect de la terre plutôt qu'une entreprise de travaux publics, mieux outillée sans doute, plus rapide dans l'exécution, mais bousculant tout et

(*) Fiat 52, $50 \mathrm{ch}$, et Saint-Chamond, $35 \mathrm{ch}$. 


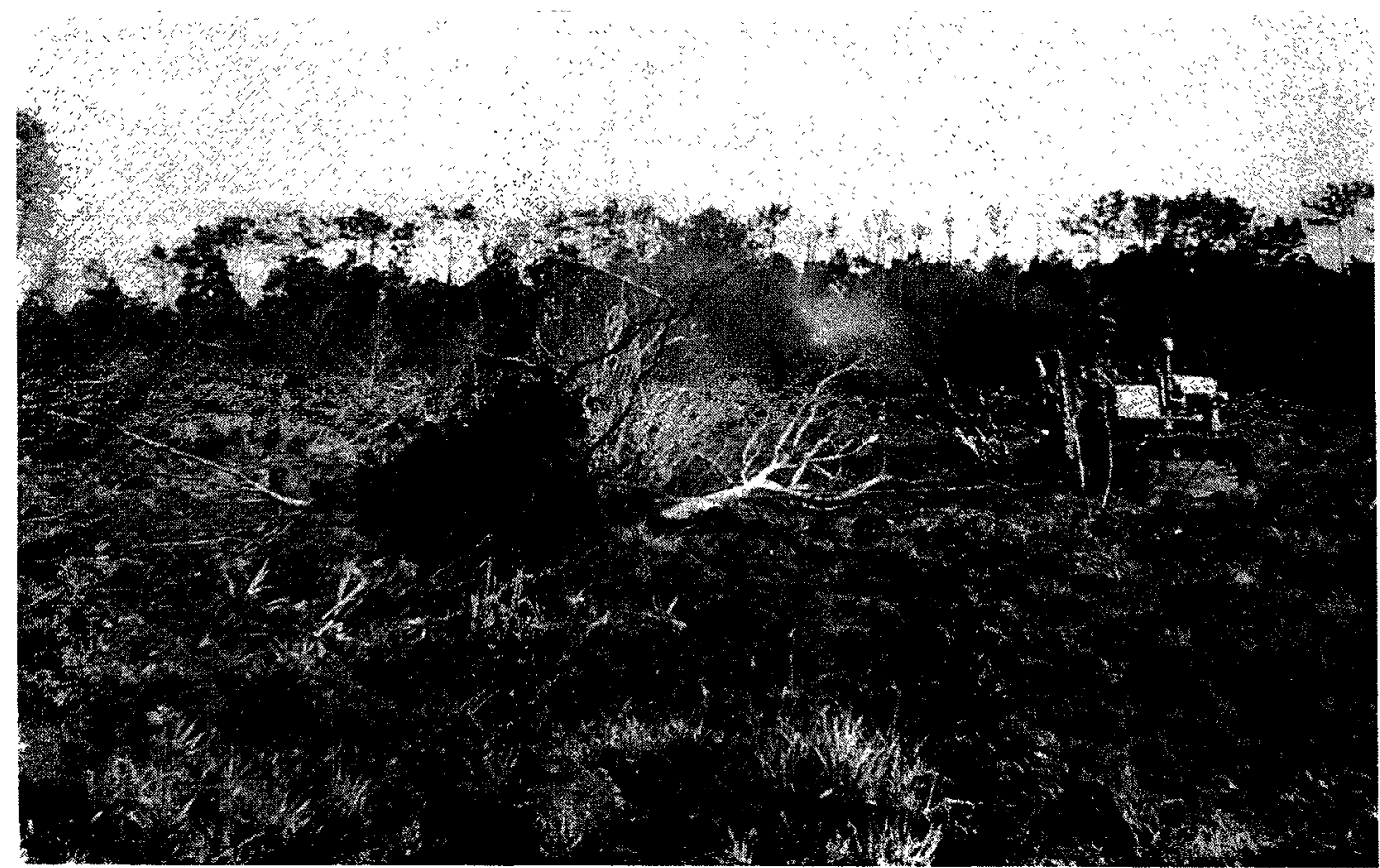

Fic. 9. - Déboisement au câble.

faisant au sol en formation le plus grand mal. Les façons nécessaires au défrichement sont en général les suivantes :

D'abord le déboisement au tracteur et au câble, parfois au treuil, avec arrachement des souches pas trop grosses; emploi d'une dessoucheuse, conçue et construite au chantier, pour l'enlèvement des grosses souches. Puis coupe du bois afin de conserver les «perches», qui, sans être liées en fascines, serviront à l'établissement des pistes de circulation. Enfin glanage des bois restant et transport par traîneaux à déchargement rapide.

La portance des terres étant passée progressivement, sous l'effet du drainage, de 200 à 400 $\mathrm{g} / \mathrm{cm}^{2}$, le déboisement est maintenant facilité par l'emploi d'un bull-dozer léger ${ }^{\star \star}$ ) qui arrache les souches au stumper et les glanes au rootrake.

Par trois ou quatre passages d'extirpateurs, en croix l'un par rapport à l'autre, on arrache toutes les petites souches, brindilles et surtout les racines. Nouveau glanage, et enlèvement ou écobuage des débris.

Dans les zones non boisées, mais à fortes têtes de chien ( 40 à $60 \mathrm{~cm}$ de haut -20 à $50 \mathrm{~cm}$ de diamètre - densité 2 à $4 \mathrm{au} \mathrm{m}^{2}$ ), on fait effectuer par le feu, lorsque le temps s'y prête, le plus

${ }^{* \star}$ Fiat 55. gros de l'ouvrage entre trois ou quatre passages successifs d'extirpateurs et de herses.

Une opération difficile, heureusement fragmentaire, a été nécéssaire pour arracher les bois fossiles enfouis à profondeur de labour : détection à la sous-soleuse, provoquant parfois l'éclatement du bois, puis enlèvement au câble, nécessitant souvent un petit terrassement manuel et l'emploi de 2 ou même 3 tracteurs pour décoller les plus grands troncs; certains avaient $28 \mathrm{~m}$ de long et $1,50 \mathrm{~m}$ de diamètre. Des centaines de

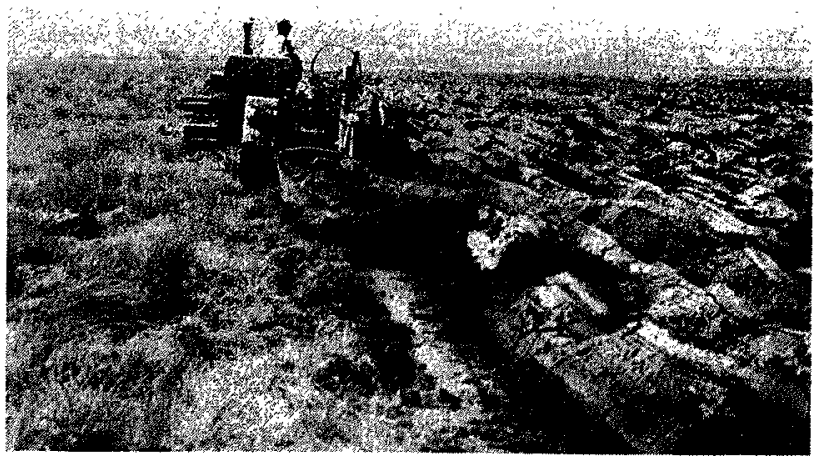

Fig. 10. - Labou à la charrue Carrière et Guyot. (mai 1950). 
troncs entiers, et des milliers de débris fossiles ont ainsi dû être retirés.

Le dessouchage et l'enlèvement des bois fossiles laissant subsister de nombreuses excavations, il a été nécessaire de les combler; d'où l'emploi de la pelle mécanique Fondeur, en travail continu et d'un mouvement constamment tournant (en forme de 8 entrecroisés), ou bien travail délicat au bull-dozer.

Le labour, d'abord exécuté à la charrue balance, a présenté de grosses difficultés dues au mauvais glissement de la tourbe le long des versoirs, pourtant longs, et même allongés par l'atelier, et par la nécessité de n'assurer qu'un retournement à $90^{\circ}$ et non à $180^{\circ}$ (ce qui eut risqué d'établir un horizon imperméable, constitué par l'épais lacis de racines, à profondeur de labour, et ainsi empêché les remontées d'eau par capillarité); par ailleurs, le film de tourbe labouré formait, par l'entrelacement des racines, une masse continue atteignant jusqu'à plusieurs centaines de mètres de long, et finissait parfois par se retourner complètement dans la raie de labour, reprenant sa position initiale.

Les terres sont ensuite ameublies au pulvíriseur à disques.

Après essais comparatifs en culture, on remplace maintenant le labour avec retournement par des grattages ou fraisages répétés, sans retournement, assurant rapidement une égale aération du sol à constituer.

Ce travail d'aération exige au moins six passages d'appareils : deux façons d'extirpateurs, travaillant à $30-35 \mathrm{~cm}$ de profondeur, perpendiculairement l'un à l'autre, deux passages de fraise rotative, deux ou trois passages de herses ou émousseuse travaillant en longs trains de 6 à 8 éléments en série.

La fraise Seaman-tiller initialement utilisée a été remplacée en mars 1953 par une fraise débroussailleuse Chevallier, sur prise de force et avec relevage hydraulique, quí, après de multiples renforcements et mises au point, donne de remarquables résultats et est d'un emploi beaucoup plus économique.

\section{Assainissement complémentaire}

Le réseau d'assainissement établi de 1948 à 1950 pour le Syndicat du Marais-Vernier constitue un réseau primaire formant des mailles de $400 \mathrm{~m}$ en moyenne de côté, mais atteignant souvent dans la partie centrale 800 et même $1.000 \mathrm{~m}$ de côté; il a été nécessaire de recouper ces grandes mailles par l'établissement d'un réseau de fossés secondaires, reprenant en général le tracé d'anciens fossés plus ou moins completement envasés.

D'autre part, la tourbe constitue un milieu colloïdal. acide, très sensible aux risques de dessiccation, pâteux ct relativement (du moins près de la surface) très imperméable. Il est donc nécessaire de lui permettre de collecter en hiver les eaux de ruissellement, puis de s'égoutter et, à l'inverse, de conserver en été une teneur en eau élevée. J'où l'obligation de recouper le terrain par un jéseau très dense de fossés et rigoles assurant un bon drainage ou une irrigation réelle.

Quel profil et quelle densité donner à ces fos-

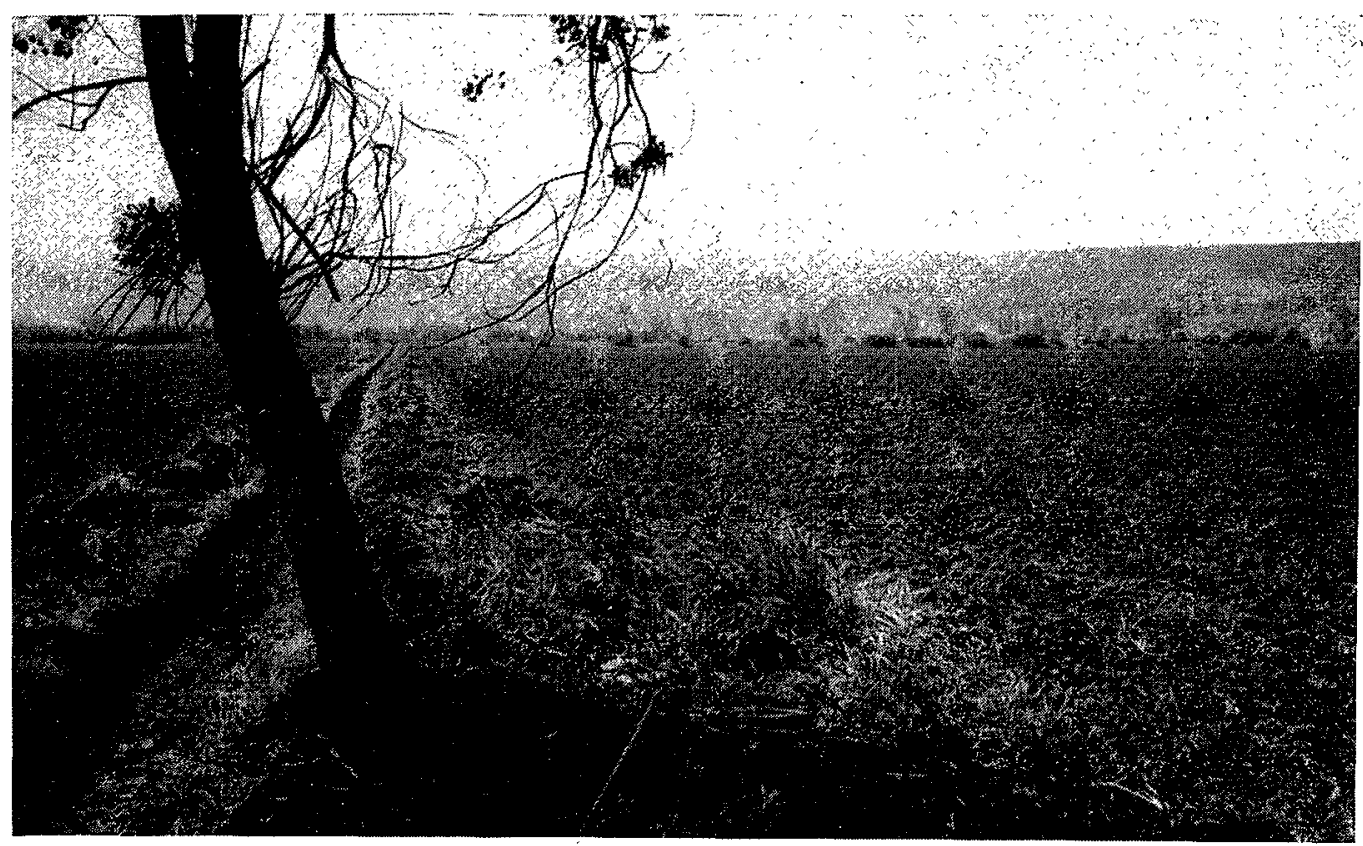

Fir, 11. - Rigole d'assainissement. 
sés? Etant donné l'hétérogénéité du marais, il est difficile de Jes fixer a priori. L'expérience hollandaise, dans des régions il est vrai un peu humides et à plus faible évaporation, indique que des fossés de 1,20 m de largeur et $1 \mathrm{~m}$ de profondeur doivent être exécutés tous les $30 \mathrm{~m}$ et qu'entre deux fossés voisins, deux petites rigoles (à $10 \mathrm{~m}$ d'espacement), de $0,20 \mathrm{~m}$ de large et $0,40 \mathrm{~m}$ de profondeur, doivent assurer l'écoulement superficiel des eaux.

Mais en Hollande il n'y a pas de bancs de tourbe profonde; on a par contre constaté au Marais-Vernier qu'il existe généralement, vers $-0,60-0,80$, une couche de tourbe fibreuse, non oxydée ni tassée et relativement perméable. L'infiltration des eaux par cette couche de tourbe est bonne; des terrains drainés à cette profondeur se sont ressuyés en une dizaine de jours en période très pluvieuse d'automne.

En ce qui concerne l'espacement des fossés et rigoles, il a fallu tenir compte de l'état initial des lieux : existence d'anciens fossés plus ou moins comblés et dont le comblement total présente d'extrêmes difficultés en terrain de tourbe (l'oxydation plus profonde, une structure différente de la matière provoquant des tassements irréguliers, maintiennent une circulation d'eau, d'où un sol plus humide, mou, vaseux, conduisant à des enfouissements de tracteurs); ces anciens fossés ont donc dû être remis en état.

Entre eux, et suivant l'état des terres, l'écartement des fossés a été en moyenne conservé à $50 \mathrm{~m}$, avec un profil de $1 \mathrm{~m}$ en gueule, $0,50 \mathrm{~m}$ au plafond et $0,75 \mathrm{~m}$ de profondeur.

Toutefois, et suivant l'état des lieux, cette distance a pu dans certains cas (de tourbe moins pâteuse - terrains de la $3^{\circ}$ tranche) être portée à $80 \mathrm{~m}$; le profil est alors plus important : $1,50 \mathrm{~m}$ en gueule, $0,75 \mathrm{~m}$ au plafond; $1 \mathrm{~m}$ de profondeur.

Ce réseau tertiaire est donc relativement hétérogène. Il n'en est pas résulté d'inconvénients pour sa création, parce que les travaux ont été faits à la main - et à la tâche.

L'essai de confection mécanique de ces rigoles a, bien entendu, été plusieurs fois tenté, au moyen de charrues à fossés et fossoyeuses diverses, tractées. Les résultats obtenus ont été décevants : ou bien, du fait de la mauvaise portance des terrains, les tracteurs ne disposent pas de la puissance nécessaire, ou bien, là où le tracteur passe, le travail exécuté est irrégulier en tous sens; le film de tourbe glisse mal sur les versoirs non appropriés des machines trouvées dans le commerce. Des machines travaillant à la profondeur voulue devraient avoir des versoirs extrêmement longs (de l'ordre de $3 \mathrm{~m}$ ), d'où un bâti adéquat, des poids et des frottements incompatibles avec l'effort de traction possible sur un tel terrain.

Une enquête approfondie effectuée dans tous les pays nordiques montre que, seuls, les terrains fermes peuvent être fossoyés au moyen de machines tractées.

Toutefois, une cureuse à fossés, à vis hélicoïdale transversale, d'origine allemande, a été essayée à deux reprises au Marais-Vernier. Montée sur tracteur semi-chenilles, elle a donné satisfaction au point fixe. Mais en marche, c'est la tenue du tracteur et sa direction qui laissent beaucoup à désirer.

Force a donc été de faire exécuter le fossoyage à la main.

$55.000 \mathrm{~m}^{3}$ de tourbe ont été ainsi extraits, tant au curage d'anciens fossés qu'à la confection des rigoles (actuellement $84.000 \mathrm{~m}$ linéaires). Ces déblais sont immédiatement régalés sur les terres voisines.

L'entretien des fossés primaires et secondaires est réalisé à la pompe suceuse.

\section{Voirie}

La mise en valeur des terres de la partie centrale du Marais-Vernier exige l'aménagement de voies d'accès à ces terrains, alors qu'actuellement les routes longent la zone tourbeuse sans la traverser.

Divers chemins ruraux ont été créés, par apport de cailloux et déblais meubles sur fascines entrecroisées reposant sur sol de tourbe profonde (4 à $7 \mathrm{~m}$ ). Ces chemins sont prolongés par de simples pistes et équipés de passerelles en bois aisément démontables, d'une force de 6 à 16 tonnes.

\section{Mise en valeur. - Amendements}

Suivant la définition, les terres défrichées se présentent comme un terrain propre, plan, aéré, sans végétation, coupé de fossés et rigoles pour l'assainissement-irrigation, et muni de pistes de circulation, passerelles et passages busés.

De cette tourbe nue il faut faire un sol de culture. La seule oxydation naturelle, due à une bonne aération, est efficace, le Iravail superficiel d'entretien, ne serait-ce que pour faire disparaitre la végétation qui s'y implante aussitôt, active cette oxydation et permet la prolifération rapide des aérobies.

Mais les analyses chimiques en montrent l'acidité ( $\mathrm{pH} 4,3$ à 6 ) et le déséquilibre, il faut les corriger. D'autre part, si ce sol est très riche en azote, bien peu en est assimilable. Enfin la tourbe est encore presque imperméable et ne permet que rarement qu'on la travaille; dis qu'il pleut le passage des tracteurs devient impossible.

Trois transformations sont donc nécessaires : - Transformation physique : rompre le colloïde de la partie superficielle pour détruire la 


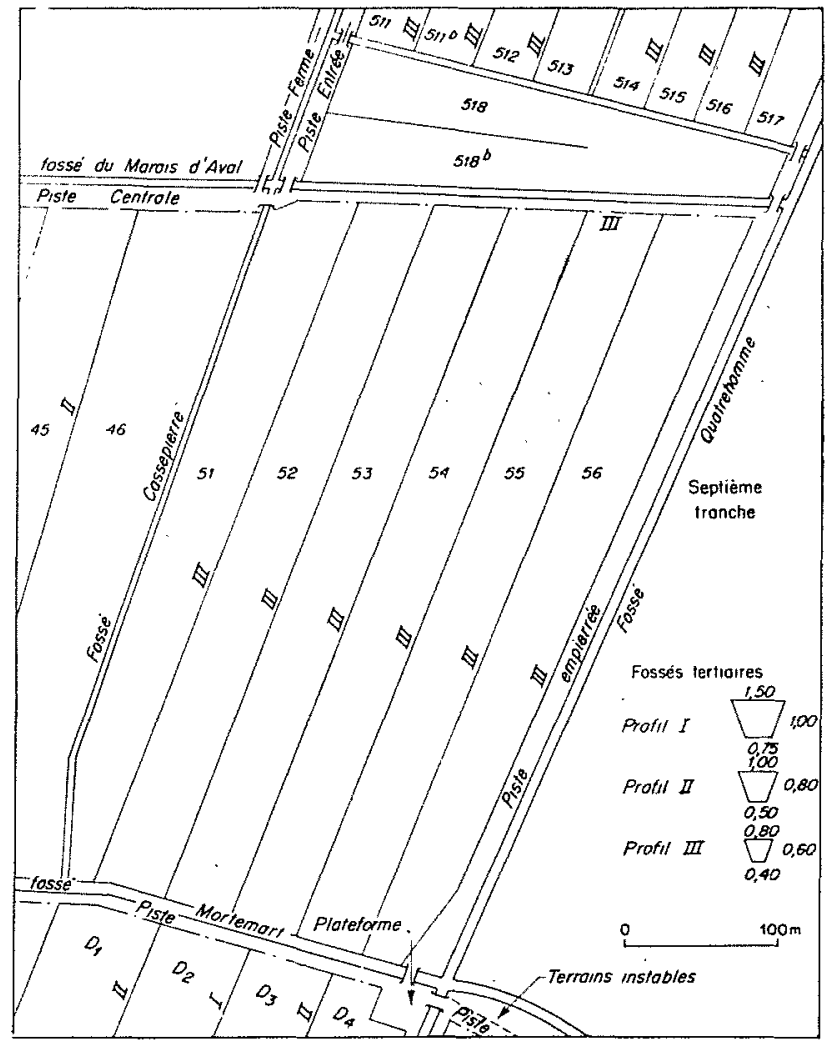

Fig. 12. - Exemple d'aménagement d'une parcelle.

nature pâteuse de la tourbe, la rendre perméable et ainsi maintenir l'aération;

- Transformation chimique par addition des éléments manquants ou en trop faible quantité, potasse et phosphate, relèvement du $\mathrm{pH}$ par apport de calcaire qui, en même temps, permettra la nitrification;

- Transformation bactériologique enfin, conséquence des modifications apportées au milieu, qui permettront la vie bactérienne utile et entretiendront cette nitrification.

Sur le plan pratique, deux opérations sont donc à faire, qui en fait se ramènent à une seule : l'épandage d'engrais et amendements avec leur enfouissement par des passages de herses.

Les engrais de base choisis pour la première tranche de 150 hectares ont été :

- L'hyperphosphate Réno $26 \%$, phosphate de Gafsa pulvérisé (tamis 300) contenant $26 \%$ d'acide phosphorique insoluble et $45 \%$ de chaux sous forme de carbonate et phosphate - soit 1 tonne/hectare;

- Le chlorure de potassium $50 \%$ - soit 250 $\mathrm{kg} /$ hectare;

- La finesse de l'hyperphosphate assure une excellente diffusion et pénétration dans le sol de la chaux dont l'effet sur le colloïde est très rapide; l'acide phosphorique, peu soluble, ne fait pratiquement sentir ses effets qu'à longue échéance, mais pour de toutes premières cultures préparatoires l'inconvénient est mineur.

L'entretien des terres a ensuite été obtenu par des scories potassiques 12/12.

Pour les terres de la deuxième tranche de travaux (200 hectares), dont la vocation immédiate semblait être la prairie, il a été jugé nécessaire d'apporter plus de chaux, ainsi que de l'acide phosphorique sous une forme plus rapidement assimilable. On y a donc épandu :

- Du calcaire broyé — à raison de $1 \mathrm{t} / \mathrm{ha}$;

- Des scories potassiques 12/12 - également à raison de $1 \mathrm{t} / \mathrm{ha}$.

Dans l'un et l'autre cas, les effets physique, chimique et bactériologique ont été tout à fait satisfaisants. Il semble pourtant qu'on puisse sans inconvénient apporter dès le début 2 ou 3 tonnes de calcaire à l'hectare.

Ơn relève cependant dans les cultures, et même, mais plus rarement, dans les prairies réalisées, des zones à carences manifestes, sans doute dues à l'hétérogénéité du sol et au manque de certains oligo-éléments. Nous pensons surtout qu'un travail régulier de ce sol nouveau, un nivellement amélioré (le maintien d'une hauteur suffisante au-dessus du niveau d'eau étant capital) et un entretien régulier en calcaire, potasse, phosphate, devraient avoir un effet très salutaire.

\section{Cultures.}

Le défrichement ayant été commencé au début de 1949, quelques parcelles ont pu être mises en première culture dès le mois d'avril 1949. En 1951-52, 135 hectares étaient exploités en avoine noire, orge, betteraves, pommes de terre et maïsfourrage.

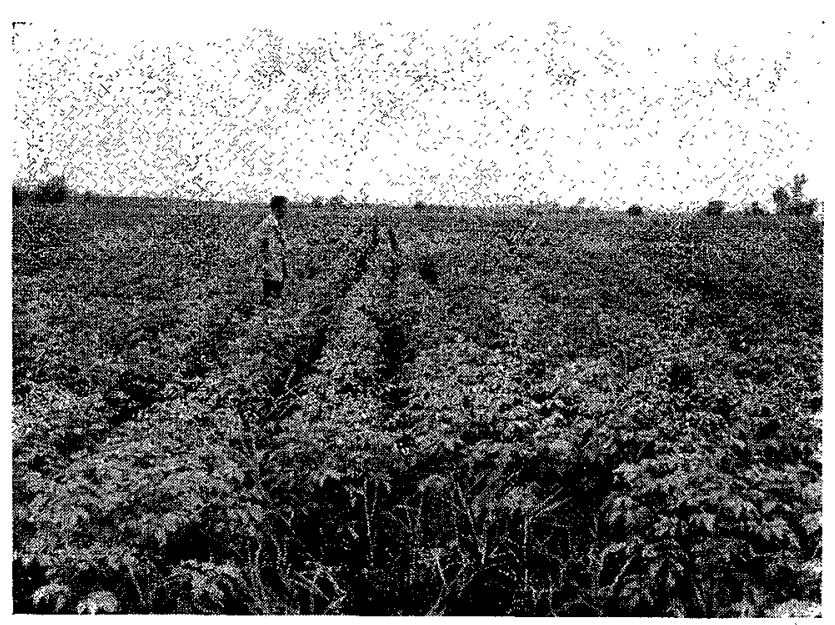

FIa. 13. - Pommes de terre (1951). 
Il résulte de ces essais de culture :

a) Que la récolte est tout à fait irrégulière certaines zones accusant des carences considérables. Le départ de la végétation est excellent et rapide; puis vient une période difficile, pendant laquelle la plante achève de vivre sur ses cotylédons et semble souffrir; les racines se forment et s'allongent cependant avec rapidité; on a bientôt un chevelu considérable; puis, prenant le dessus, la plante croît vite et bien, avec presque trop de végétation (excès d'azote), cependant que le fruit, très irrégulier, donne en définitive une récolte moyenne;

b) Que la culture de ces terres est difficile : tant que le sol n'est pas «fait », il est presque impossible de pénétrer dans les pièces au début du printemps pour les préparer et les ensemencer. Si les binages se font bien (période de sécheresse), par contre la récolte est très sujette aux intempéries; s'il pleut beaucoup, le passage de la moissonneuse-batteuse est compromis, la machine, un peu lourde, travaille très irrégulièrement. La récolte de tubercules et de racines, au début de l'automne, s'avère complètement liée au temps. Surtout le charroi de denrées lourdes devient presque impossible dès qu'il pleut, sur des pistes rendues rapidement impraticables du fait des ornieres profondes qu'y laissent les engins à roues;

c) Bien entendu, tout travail d'hiver est à peu près inconcevable;

d) Aucune verse, aucune maladie particulière n'ont été constatées dans ces terres.

La culture au Marais-Vernier semble donc peu conseillée, sinon saisonnière (maraîchère), et seulement sur certaines pièces de terre d'accès très aisé, ce qui n'est pas en général le cas dans la partie centrale.

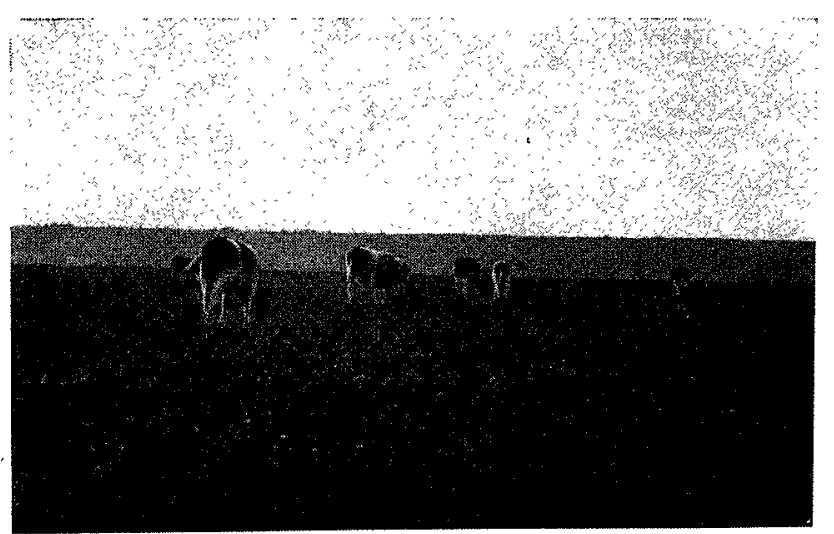

FIG. 14. - Les nouvelles prairies.

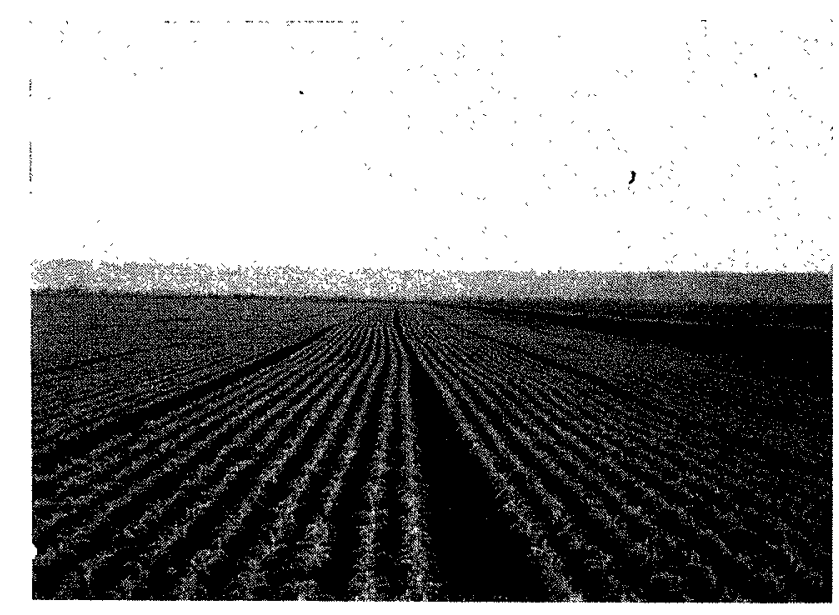

FIG. 15. - Maïs (1951).

Le problème des transports se pose d'une façon plus aiguë encore que dans n'importe quelle autre exploitation agricole.

Les conditions de temps, essentiellement variables, sont particulièrement sensibles sur ces terres, tout au moins pendant les premières années.

Enfin une meilleure couverture du sol ne sera obtenue qu'avec une végétation permanente sous forme de prairie, alors que les binages répétés et la couverture incomplète risquent, en période de grande sécheresse, de favoriser la coagulation de l'humus, à laquelle certaines tourbes sont spécialement sensibles.

Pour ces raisons, il a été décidé de renoncer au maximum $\dot{a}$ la culture, et de ne travailler annuellement qu'une faible surface destinée à fournir la nourriture complémentaire du bétail.

\section{Prairies.}

A la fin de l'été 1951, une centaine d'hectares de terres de la partie centrale du Marais-Vernier, en région de tourbe profonde, à têtes de chien et taillis, étaient totalement défrichés, labourés, ameublis et assainis par de nombreuses rigoles. L'épandage d'engrais ( $1 \mathrm{t}$. scories potassiques et 0 à 1 tonne de calcaire broyé à l'hectare) fut fait aussitôt, puis l'ensemencement en herbages avec le mélange de semences conseillé par M. Hénin, Directeur du Laboratoire des Plantes Fourragères de Rouen, éminent spécialiste des herbages normands, soit $35 \mathrm{~kg}$ de graines à l'hectare.

La levée avant l'hiver (bien que l'ensemencement ne fut achevé que le 20 octobre) fut excellente, l'effet du froid, il est vrai peu rigoureux, de cet hiver, fut apparemment nul.

Une première fauche générale sur les 100 hectares de prairie fut faite en mai-début juin, puis une seconde sur 40 hectares environ à fin juil- 




Fig. 16. - Les étables (août 1953).

let, tandis que le bétail commençait à pâturer successivement, derrière clôtures électriques, l'herbe des différentes parcelles. C'est ainsi que, dès la première année, certaines parcelles ont été fauchées deux fois et pâturées trois fois; d'autres fauchées une fois et pâturées quatre fois: le bétail put rester sur place jusqu'au 20 décembre, avec apport de bottes de foin complémentaire depuis le 8 décembre seuiement.

Quelle a été la récolte de foin? On l'estime en moyenne de 6 à 7 tonnes, en sec, d'un mélange excellent où dominait en début de saison le ray-grass, et à l'automne un magnifique trèfle blanc.

Faut-il en conclure que ces herbages sont parfaits? Non, sans doute, parce qu'ayant encore quelques irrégularités, en particulier sur les bordures; quelques touffes de joncs, et quelques carex y poussent encore de loin en loin et par endroits beaucoup de houlque laineuse; d'autre part, le trèfle violet et le dactyle ont mal prospéré. Ceci tient pour une grande part au manque de calcaire de ce sol; des amendements devront régulièrement y être apportés, les joncs détruits, etc...

Quoi qu'il en soit, et tels qu'ils étaient en 1953, ces herbages sont estimés pouvoir nourrir 3 bêtes à l'hectare, ce qui leur donne dès maintenant une valeur foncière de plus de $350.000 \mathrm{fr}$. l'hectare.

Effectivement, un premier troupeau d'une soixantaine de vaches hollandaises a commencé à pâturer ces prés vers le $1^{\text {er }}$ juillet 1952 , dont une trentaine de laitières; ce troupeau a été doublé d'un troupeau normand équivalent, dès le mois d'août, et en outre, une trentaine de bœufs du Charolais ont été pris en pension d'août à fin décembre.

110 hectares de terres précédemment cultivées $(1950,51$ et 52$)$ ont été ensemencées en herbages à l'automne 1952 dans les mêmes conditions que les premiers, quoique sans nouvel ap- port de scories ou de calcaire; ceux-ci n'ont été épandus qu'en janvier-février 1953 sur les herbages faits.

350 hectares enfin ont été ensemencés en herbes en 1953 et 1954 .

220 hectares $\left(7^{\circ}\right.$ et $8^{\circ}$ tranches) sont encore à défricher et mettre en prairie au cours des prochains mois.

\section{La Ferme.}

L'évolution de l'exploitation de la partie centrale du Marais-Vernier, nouvellement assainic et défrichée, semble ainsi achevée. Des 400 hectares constituant les trois premières tranches de travaux, 350 seront exploités pour l'herbe. Ceci implique donc une spécialisation des bâtiments d'exploitation à construire.

On s'est décidé finalement pour la construction d'étables classiques, soit 6 étables à 52 postes chacune, à stalles courtes, avec monorail d'alimentation, tapis de caoutchouc sous les bètes, transporteur mécanique du fumier vers des fosses couvertes; une laiterie est installée. La traite mécanique se fait soit en stations mobiles sur les herbages, soit à l'étable pendant l'hiver.

Un puits a été foré à proximité de la ferme; des hangars provisoires seront remplacés par un atelier de réparation et des granges; trois maisons d'habitation sont construites.

Tous ces bâtiments, en matériaux très simples, bien conçus et étudiés, donnent son caractère définitif à cette partie du Marais-Vernier.

\section{Les résultats}

Il est peut-être trop tôt pour donner comme définitifs les résultats actuels. Ceux-ci sont déjà L rès satisfaisants, et nous pensons qu'ils seront notablement accrus par de multiples petits travaux d'amélioration et surtout par un entretien



Fig. 17. - Première étable achevée (août 1953). 
attentif et soigneux, entretien des fossés et rigoles, entretien aussi des pistes de circulation, mais surtout entretien des terres elles-mêmes par un apport régulier de calcaire broyé, si possible magnésien; un apport aussi, périodique, de scories potassiques. II doit en résulter une bonification générale des terres se traduisant par la qualité de la végétation et sa quantité (on doit arriver au moins à 15 tonnes d'excellent foin sec à l'hectare), d'où la possibilité de nourrir l'importance des travaux exécutés de 6.000 à $16,000 \mathrm{fr}$. l'hectare.

La Compagnie des Techniques Hydrauliques et Agricoles a conseillć et dirigé ces travaux depuis leur origine.

La mise en valeur agricole du Marais-Vernier a constitué, pour la France, une expérience intéressante puisque plusieurs centaines de milliers d'hectares de terrains tourbeux, soit près de $2 \%$



Fig. 18. - Le résultat : magnifique prairie avec prédominance de trèfle.

complètement trois bêtes de choix à l'hectare, justifiant une valeur foncière supérieure à $350.000 \mathrm{fr}$.

Ces travaux ont été réalisés, sous le contrôle du Génie Rural de l'Eure, par les associalions syndicales de propriétaires, avec des subventions de l'Etat, soit $60 \%$ du prix de tous les travaux d'assainissement, et des prêts de la Caisse Nationale de Crédit Agricole remboursables en 20 ou 25 ans, et en moyenne de 2 à $3 \%$ d'intérêt.

L'annuité à l'hectare à payer par les propriétaires des terres mises en valeur varie suivant de la surface cultivable française, sonl encore inexploités.

Etudiée et réalisée pendant les années dilficiles qui ont inmédiatement suivi la fin de la guerre, au cours desquelles les échanges internationaux d'information et de documentation ont été très réduits, elle constitue une entreprise particulièrement intéressante parce qu'elle a permis d'étudier quelques questions se rapportant au domaine peu connu de la tourbe, et aussi parce qu'elle a donné des résultats satisfaisants dont la reproduction ailleurs peut maintenant être entreprise avec certitude de succis. 